\title{
One axiom to rule them all: An axiomatization of quantiles
}

\author{
Tolulope Fadina* $\quad$ Peng Liu ${ }^{\dagger} \quad$ Ruodu Wang ${ }^{\ddagger}$
}

October 17, 2021

\begin{abstract}
We offer an axiomatic characterization of quantiles through only one axiom. Among all real-valued mappings on a general set of distributions, left quantiles are the only ones satisfying left-ordinal covariance, meaning that they commute with increasing left-continuous transforms; the case of right quantiles is analogous. Other convenient properties of quantiles, monotonicity in particular, follow from this axiom. In banking and insurance, quantiles are known as Value-at-Risk (VaR), a standard regulatory risk measure. Thus, we obtain an axiomatization of VaR with only one axiom among law-based risk measures. We further show that VaR can be alternatively characterized via the axiom of locality, plus four standard axioms relevant in financial risk management, namely, monotonicity, normalization, cash additivity, and semicontinuity.
\end{abstract}

Key-words: ordinal covariance; locality; monotonicity; risk measures; Value-at-Risk

\section{Introduction}

Quantiles are prominent objects in statistics, decision theory, optimization, machine learning, and finance, and they have been widely applied in the natural and social sciences as well as engineering through quantile regression and quantile optimization; see e.g., Koenker and Hallock (2001). In this paper, we offer an axiomatization of quantiles among all mappings on a general set of distributions via only one property. We first explain our main result.

Let $\mathcal{X}$ be a set of random variables in an atomless probability space $(\Omega, \mathcal{F}, \mathbb{P})$ containing all bounded random variables. The left quantile at probability level $p \in(0,1]$ is defined as

$$
Q_{p}(X)=\inf \{x \in \mathbb{R}: \mathbb{P}(X \leqslant x) \geqslant p\}, \quad X \in \mathcal{X},
$$

\footnotetext{
*Department of Mathematical Sciences, University of Essex, UK. Email: t.fadina@essex.ac.uk

${ }^{\dagger}$ Department of Mathematical Sciences, University of Essex, UK. Email: peng.liu@essex.ac.uk

${ }^{\ddagger}$ Department of Statistics and Actuarial Science, University of Waterloo, Canada. Email: wang@uwaterloo.ca
} 
where $\inf \emptyset=\infty$. We consider law-based mappings $\mathcal{R}$ on $\mathcal{X}$, meaning that $\mathcal{R}(X)$ is determined by the distribution of $X$. Throughout, terms like "increasing" are in the non-strict sense.

Theorem 1. For a law-based mapping $\mathcal{R}: \mathcal{X} \rightarrow \mathbb{R}$, the following are equivalent:

(i) $\mathcal{R} \circ \phi=\phi \circ \mathcal{R}$ for all increasing left-continuous functions $\phi: \mathbb{R} \rightarrow \mathbb{R}$;

(ii) $\mathcal{R}=Q_{p}$ for some $p \in(0,1]$.

The property in Theorem 1 (i) will be called left-ordinal covariance, and $\mathcal{R} \circ \phi=\phi \circ \mathcal{R}$ means that $\mathcal{R}(\phi(X))=\phi(\mathcal{R}(X))$ for all $X \in \mathcal{X}$ such that $\phi(X)$ remains in $\mathcal{X}$. An alternative version of Theorem 1, including right quantiles, is formulated as Theorem 2 in Section 3.

The interpretation of left-ordinal covariance in decision making is roughly the same as ordinal covariance: For an index which quantifies random objects, a possibly non-linear scale change in the random outcomes gives rise to the same scale change on the index; see e.g., Chambers (2007) in the context of utility aggregation.

Theorem 1 is an extension of Chambers (2009), who showed that the left quantile is the only function that satisfies the three axioms of monotonicity, lower semicontinuity, and ordinal covariance (commuting with strictly increasing and continuous functions); see Section 2 for details. Contrasting Theorem 1 with that result, our left-ordinal covariance is slightly stronger than ordinal covariance, and monotonicity and lower semicontinuity are not imposed. Moreover, results of Chambers (2009) are obtained on either $\mathcal{X}=L^{\infty}$ (the set of essentially bounds random variables) or $\mathcal{X}=L^{0}$ (the set of all random variables) whereas our result holds on any domain $\mathcal{X}$ with $L^{\infty} \subseteq \mathcal{X} \subseteq L^{0}$.

Despite the obvious similarity, our extension of Chambers (2009) is by no means straightforward. Enlarging the set of functions that commute with $\mathcal{R}$ makes the corresponding covariance property stronger, but choosing such an enlargement requires subtle sophistication. For instance, one may be tempted to require $\mathcal{R}$ to commute with all increasing functions, but such $\mathcal{R}$ does not exist; see Example 1 below. Therefore, extra care has to be taken when formulating the set which $\mathcal{R}$ commutes with, and this may partially explain why the concise characterization in Theorem 1 was not found in the literature.

Example 1. There is no such $\mathcal{R}: L^{\infty} \rightarrow \mathbb{R}$ satisfying $\mathcal{R} \circ \phi=\phi \circ \mathcal{R}$ for all increasing function $\phi$. To see this, suppose otherwise. Take any continuously distributed random variable $X \in L^{\infty}$ and denote by $a=\mathcal{R}(X)$. Let $\phi_{1}(x)=\mathbb{1}_{\{x \geqslant a\}}$ and $\phi_{2}(x)=\mathbb{1}_{\{x>a\}}$, where $\mathbb{1}$ is the indicator function. Note that $\phi_{1}(X)=\phi_{2}(X)$ almost surely (and thus they are equal in $L^{\infty}$ ). A contradiction arises as $\mathcal{R}\left(\phi_{1}(X)\right)=\phi_{1}(\mathcal{R}(X))=1>0=\phi_{2}(\mathcal{R}(X))=\mathcal{R}\left(\phi_{2}(X)\right)=\mathcal{R}\left(\phi_{1}(X)\right)$.

In Section 4 we discuss in more detail whether the set of transforms in Theorem 1 can be made smaller without invalidating the characterization result. If we concentrate on compactly 
supported distributions, there is a possible slight relaxation in Proposition 1, but such relaxations are not harmless in general, as illustrated by means of two counter-examples. Moreover, in Proposition 2, we improve a result of Chambers (2009) on unbounded distributions by relaxing the property of strong ordinal covariance.

Since quantiles are characterized by left-ordinal covariance alone, all the other nice properties of quantiles, such as monotonicity, cash additivity, positive homogeneity, continuity, comonotonic additivity (see Föllmer and Schied (2016)), elicitability (see Gneiting (2011)), and tail relevance (see Liu and Wang (2021)) are obtained for free. In particular, it may be surprising that monotonicity is implied, noting that it does not follow from ordinal covariance of Chambers (2009). An observation in a similar fashion is made by Wang and Wei (2020), in which a comonotonic-additive, elicitable and uniformly continuous mapping on $L^{\infty}$ is necessarily monotone (either increasing or decreasing), although no single one of these properties implies monotonicity by itself.

We proceed to show in Theorem 3 that quantiles can be characterized via locality, which roughly means that the output value of the mapping depends on the input distribution only through its behaviour around the output value itself. This property may be seen as a numerical counterpart of the pivotal monotonicity axiom of Rostek (2010). In particular, a law-based mapping is normalized, monotone, semicontinuous, cash additive and local if and only if it is a quantile; see Section 5 for details. This result is built on a recent characterization of $\Lambda$-quantiles by Bellini and Peri (2021) using locality; $\Lambda$-quantile are a generalization of VaR proposed by Frittelli et al. (2014).

Our result gives a simple axiomatic foundation for quantiles, and it may be useful in many other contexts, as quantiles are a useful alternative to the standard utility theory in decision making. Preferences induced by quantiles are axiomatized by Rostek (2010), and Embrechts et al. (2018) studied collaborative and competitive equilibria for quantile agents. de Castro and Galvao (2019) developed a dynamic model for a rational quantile maximizer, and de Castro et al. (2020) provided empirical evidence for decision making with quantile optimization.

In financial risk management, quantiles are known as Value-at-Risk (VaR), the dominating risk measure in banking and insurance over the past two decades. Although Expected Shortfall (ES) is proposed to replace VaR in the recent Basel Accords, VaR is still widely applied in regulatory capital calculation, decision making, performance analysis, and backtesting. We refer to McNeil et al. (2015) for a general treatment on VaR and other risk measures. Because of the practical importance of VaR, there are a few other sets of axioms for VaR, or quantiles, in addition to Chambers (2009). With some other standard properties including monotonicity, VaR is characterized via elicitability and comonotonic additivity by Kou and Peng (2016), via 
surplus invariance by He and Peng (2018), and via elicitability and tail relevance by Liu and Wang (2021). On the other hand, ES is axiomatized by Wang and Zitikis (2021) with a property reflecting portfolio diversification. In each result above, a class of risk measures is characterized by at least three properties, whereas Theorem 1 axiomatizes VaR using only one.

For the rest of the paper, we conveniently formulate all mappings with domains being sets of distributions instead of random variables. Although we have focused on left quantiles in the Introduction, results on right quantiles are analogous to those on left quantiles, and they will be presented in a symmetric manner.

\section{Preliminaries}

Let $\mathcal{M}_{0}$ be the set all distributions on $\mathbb{R}$ and $\mathcal{M}_{c}$ be the set of all compactly supported distributions in $\mathcal{M}_{0}$. Throughout the paper, $\mathcal{M}$ is the domain of a mapping of interest, satisfying $\mathcal{M}_{c} \subseteq \mathcal{M} \subseteq \mathcal{M}_{0}$. Distributions in $\mathcal{M}$ will be identified with their cdfs. For a cdf $F \in \mathcal{M}$, we define its (left) quantile as

$$
F^{-1}(t)=\inf \{x \in \mathbb{R}: F(x) \geqslant t\}, \quad t \in(0,1]
$$

and its right quantile as

$$
F_{+}^{-1}(t)=\inf \{x \in \mathbb{R}: F(x)>t\}, \quad t \in[0,1) .
$$

Denote by $\mathcal{G}^{L}$ the set of all increasing left-continuous functions $\phi: \mathbb{R} \rightarrow \mathbb{R}$ and by $\mathcal{G}^{R}$ the set of all increasing right-continuous functions $\phi: \mathbb{R} \rightarrow \mathbb{R}$. For notational convenience, we extend the domain of $F \in \mathcal{M}$ to $[-\infty, \infty]$ by letting $F(-\infty)=0$ and $F(\infty)=1$.

For an increasing function $\phi$, the shape transform $T^{[\phi]}: \mathcal{M} \rightarrow \mathcal{M}$ is defined as a mapping from the distribution of a random variable $X$ to the distribution of $\phi(X)$. In particular,

(a) for $\phi \in \mathcal{G}^{L}, T^{[\phi]}(F)(x)=F\left(\phi_{+}^{-1}(x)\right), x \in \mathbb{R}$, where $\phi_{+}^{-1}(x)=\sup \{y \in \mathbb{R}: \phi(y) \leqslant x\}$;

(b) for $\phi \in \mathcal{G}^{R}, T^{[\phi]}(F)(x-)=F\left(\phi^{-1}(x)-\right), x \in \mathbb{R}$, where $\phi^{-1}(x)=\inf \{y \in \mathbb{R}: \phi(y) \geqslant x\}$.

The convention here is $\inf \emptyset=\infty$ and $\sup \emptyset=-\infty$, and for any function $f, f(x-)=\lim _{y \uparrow x} f(x)$ and $f(x+)=\lim _{y \downarrow x} f(x)$ as usual. In the context of distributional transforms, which are mappings from $\mathcal{M}$ to $\mathcal{M}$, Liu et al. (2021) showed that shape transforms can be used to characterize probability distortions; similarly, for mappings from $\mathcal{M} \rightarrow \mathbb{R}$, shape transforms can be used to characterize quantiles, as in Chambers (2009) and this paper.

In the remainder of the paper, different from Section 1, we will conveniently treat law-based mappings as functions from $\mathcal{M}$ to $\mathbb{R}$. We first define left- or right-ordinal covariance, the most important property in this paper. 
Definition 1. A mapping $\rho: \mathcal{M} \rightarrow \mathbb{R}$ satisfies left-ordinal covariance (resp. right-ordinal covariance) if $\rho \circ T^{[\phi]}=\phi \circ \rho$ for all $\phi \in \mathcal{G}^{L}\left(\right.$ resp. $\left.\phi \in \mathcal{G}^{R}\right)$.

Here, $\rho \circ T^{[\phi]}=\phi \circ \rho$ means that $\rho\left(T^{[\phi]}(F)\right)=\phi(\rho(F))$ for all $F \in \mathcal{M}$ such that $T^{[\phi]}(F) \in \mathcal{M}$. Left- or right-ordinal covariance is stronger than ordinal covariance assumed by Chambers (2009). We explain the three axioms of Chambers (2009) below.

Definition 2. For a mapping $\rho: \mathcal{M} \rightarrow \mathbb{R}$,

(i) $\rho$ is monotone if $\rho(F) \leqslant \rho(G)$ whenever $F \leqslant$ st $G$ and $F, G \in \mathcal{M}$, where $F \leqslant$ st $G$ means $F(x) \geqslant G(x)$ for all $x \in \mathbb{R}$;

(ii) $\rho$ is lower semicontinuous (resp. upper semicontinuous) if $\rho(F) \leqslant \alpha$ (resp. $\rho(F) \geqslant \alpha$ ) whenever $\left\{F_{n}\right\}_{n \in \mathbb{N}} \subseteq \mathcal{M}$ with $\rho\left(F_{n}\right) \leqslant \alpha$ (resp. $\rho\left(F_{n}\right) \geqslant \alpha$ ) converges weakly to $F \in \mathcal{M}$;

(iii) $\rho$ satisfies ordinal covariance if $\rho \circ T^{[\phi]}=\phi \circ \rho$ for all $\phi \in \mathcal{G}^{*}$, where $\mathcal{G}^{*}$ is the set of all strictly increasing and continuous functions $\phi$ satisfying $\phi(\mathbb{R})=\mathbb{R}$.

Since $\mathcal{G}^{*} \subsetneq \mathcal{G}^{L} \cap \mathcal{G}^{R}$, it is clear that left- or right-ordinal covariance implies ordinal covariance. As the main result of Chambers (2009, Theorem 1), for $\mathcal{M}=\mathcal{M}_{c}$, monotonicity, lower semicontinuity and ordinal covariance together are sufficient and necessary for $\rho$ to be a left quantile, that is, $\rho(F)=F^{-1}(p)$ on $\mathcal{M}_{c}$ for some $p \in(0,1]$. Chambers (2009) imposed a slightly stronger condition for a similar result on $\mathcal{M}=\mathcal{M}_{0}$, and we will see in Section 4 that this stronger condition can be relaxed.

In contrast to the three axioms in Definition 2, we will show that left-ordinal covariance alone characterizes the left quantile, and monotonicity and lower semicontinuity are thereby consequences instead of assumptions. Moreover, our characterization readily carries through to any domain $\mathcal{M}$ satisfying $\mathcal{M}_{c} \subseteq \mathcal{M} \subseteq \mathcal{M}_{0}$, whereas the proofs in Chambers (2009) cannot be easily extended to the setting of an arbitrary domain without strengthening ordinal covariance, since these techniques involve approximations of distributions and taking a limit.

\section{Left-ordinal covariance axiomatizes left quantiles}

In this section, we present our main result on the characterization of quantiles based on left- or right-ordinal covariance. Theorem 1 in the Introduction follows directly from Theorem 2 by translating results on $\mathcal{M}$ to those on $\mathcal{X}$.

Theorem 2. Let $\rho: \mathcal{M} \rightarrow \mathbb{R}$ where $\mathcal{M}_{c} \subseteq \mathcal{M} \subseteq \mathcal{M}_{0}$. We have

(i) $\rho$ satisfies left-ordinal covariance if and only if there exists $p \in(0,1]$ such that $\rho(F)=$ $F^{-1}(p)$ for all $F \in \mathcal{M}$; 
(ii) $\rho$ satisfies right-ordinal covariance if and only if there exists $p \in[0,1)$ such that $\rho(F)=$ $F_{+}^{-1}(p)$ for all $F \in \mathcal{M}$.

Proof. (i) To show the "if" part, note that for $p \in(0,1]$ and $\phi \in \mathcal{G}^{L}$,

$$
\begin{aligned}
\rho\left(T^{[\phi]}(F)\right) & =\inf \left\{x \in \mathbb{R}: F\left(\phi_{+}^{-1}(x)\right) \geqslant p\right\} \\
& =\inf \left\{x \in \mathbb{R}: \phi_{+}^{-1}(x) \geqslant F^{-1}(p)\right\} \\
& =\inf \left\{x \in \mathbb{R}: x \geqslant \phi\left(F^{-1}(p)\right)\right\}=\phi(\rho(F)) .
\end{aligned}
$$

Note that in the above if $F^{-1}(1)=\infty$, then $p=1$ is excluded as $\rho$ is real-valued. We next focus on the "only if" part. We will first prove the result on $\mathcal{M}_{c}$ and then extend it to $\mathcal{M}$. For any $F \in \mathcal{M}_{c}$, let

$$
\phi(x)=\left\{\begin{array}{lc}
F_{+}^{-1}(0), & x \leqslant 0 \\
F^{-1}(x), & 0<x \leqslant 1 \\
F^{-1}(1), & x>1 .
\end{array}\right.
$$

Then we have $\phi \in \mathcal{G}^{L}$ and $T^{[\phi]}\left(F_{U}\right)=F$, where $F_{U}$ is uniform on $[0,1]$. Hence,

$$
\rho(F)=\rho\left(T^{[\phi]}\left(F_{U}\right)\right)=\phi\left(\rho\left(F_{U}\right)\right) .
$$

Combination of (3) and (4) yields that for $F \in \mathcal{M}_{c}$,

$$
\rho(F)=\left\{\begin{array}{cc}
F^{-1}\left(1 \wedge \rho\left(F_{U}\right)\right), & \rho\left(F_{U}\right)>0 \\
F_{+}^{-1}(0), & \rho\left(F_{U}\right) \leqslant 0 .
\end{array}\right.
$$

The proof on $\mathcal{M}_{c}$ is complete if we show $\rho\left(F_{U}\right)>0$. We assume by contradiction that $\rho\left(F_{U}\right) \leqslant 0$. Then $\rho(F)=F_{+}^{-1}(0)$ for all $F \in \mathcal{M}_{c}$. For $\varphi(x)=x \mathbb{1}_{\{x \leqslant 0\}}+(1+x) \mathbb{1}_{\{x>0\}} \in \mathcal{G}^{L}$, we have

$$
\rho\left(T^{[\varphi]}\left(F_{U}\right)\right)=\left(T^{[\varphi]}\left(F_{U}\right)\right)_{+}^{-1}(0)=\varphi(0+)=1 .
$$

Moreover, it follows from left-ordinal covariance that

$$
\rho\left(T^{[\varphi]}\left(F_{U}\right)\right)=\varphi\left(\rho\left(F_{U}\right)\right)=\rho\left(F_{U}\right)<1=\rho\left(T^{[\varphi]}\left(F_{U}\right)\right),
$$

which is a contradiction. Hence, $\rho\left(F_{U}\right)>0$, and with $p=1 \wedge \rho\left(F_{U}\right) \in(0,1]$,

$$
\rho(F)=F^{-1}(p), F \in \mathcal{M}_{c}
$$

Next we extend (5) to general $\mathcal{M}$. Let $\phi_{0}(x)=\frac{1}{2}+\frac{1}{\pi} \arctan (x), x \in \mathbb{R}$. For $F \in \mathcal{M} \backslash \mathcal{M}_{c}$, $T^{\left[\phi_{0}\right]}(F) \in \mathcal{M}_{c}$. It follows from (5) that

$$
\rho\left(T^{\left[\phi_{0}\right]}(F)\right)=\phi_{0}\left(F^{-1}(p)\right) .
$$


Moreover, by left-ordinal covariance we have $\rho\left(T^{\left[\phi_{0}\right]}(F)\right)=\phi_{0}(\rho(F))$. Since $\phi_{0}$ is strictly increasing, we have $\rho(F)=F^{-1}(p)$. This completes the proof of (i).

(ii) The proof is similar to (i), and we mention slight differences. To show the "if" part, for $p \in[0,1)$ and $\phi \in \mathcal{G}^{R}$, we have

$$
\begin{aligned}
\rho\left(T^{[\phi]}(F)\right) & =\sup \left\{x \in \mathbb{R}: F\left(\phi^{-1}(x)-\right) \leqslant p\right\} \\
& =\sup \left\{x \in \mathbb{R}: \phi^{-1}(x) \leqslant F_{+}^{-1}(p)\right\} \\
& =\sup \left\{x \in \mathbb{R}: x \leqslant \phi\left(F_{+}^{-1}(p)\right)\right\}=\phi(\rho(F)) .
\end{aligned}
$$

In the above if $F_{+}^{-1}(0)=-\infty$, then $p=0$ is excluded. We next prove the "only if" part. For any $F \in \mathcal{M}_{c}$, let

$$
\phi(x)=\left\{\begin{array}{lc}
F_{+}^{-1}(0), & x<0 \\
F_{+}^{-1}(x), & 0 \leqslant x<1 \\
F^{-1}(1), & x \geqslant 1 .
\end{array}\right.
$$

Then we have $\phi \in \mathcal{G}^{R}$ and $T^{[\phi]}\left(F_{U}\right)=F$. It follows that $\rho(F)=\rho\left(T^{[\phi]}\left(F_{U}\right)\right)=\phi\left(\rho\left(F_{U}\right)\right)$, which, combined with (6), implies that for $F \in \mathcal{M}_{c}$,

$$
\rho(F)=\left\{\begin{array}{cc}
F^{-1}\left(0 \vee \rho\left(F_{U}\right)\right), & \rho\left(F_{U}\right)<1 \\
F^{-1}(1), & \rho\left(F_{U}\right) \geqslant 1 .
\end{array}\right.
$$

Analogously to (i), by choosing $\varphi: x \mapsto x \mathbb{1}_{\{x<1\}}+(1+x) \mathbb{1}_{\{x \geqslant 1\}} \in \mathcal{G}^{R}$, we can show that $\rho\left(F_{U}\right)<1$. This completes the proof on $\mathcal{M}_{c}$. Using $\phi_{0}$ defined in (i), we can extend the result on $\mathcal{M}_{c}$ to $\mathcal{M}$.

A useful fact in the proof of Theorem 2 is $\mathcal{M}_{c}=\left\{T^{[\phi]}\left(F_{U}\right): \phi \in \mathcal{G}^{L}\right\}$ for the uniform distribution $F_{U}$ on $[0,1]$. In other words, $\mathcal{M}_{c}$ can be generated from $F_{U}$ by shape transforms $T^{[\phi]}$ with $\phi \in \mathcal{G}^{L}$. Then left-ordinal covariance allows us to derive $\rho(F)$ for all $F \in \mathcal{M}_{c}$ in terms of the quantile of $F$ and the value of $\rho\left(F_{U}\right)$ as in (4). Note that $\mathcal{M}_{c}$ cannot be generated from a distribution by shape transforms $T^{[\phi]}$ with $\phi$ being strictly increasing and continuous, and thus (4) cannot be derived from ordinal covariance. This sheds some light on why we can skip continuity and monotonicity but Chambers (2009) needed these properties.

Remark 1. Note that $\rho$ is assumed to be real-valued on $\mathcal{M}$ in Theorem 2. The value $p=1$ in (i) needs to be excluded if $\mathcal{M}$ contains $F$ such that $F^{-1}(1)=\infty$. The value $p=0$ in (ii) needs to be excluded if $\mathcal{M}$ contains $F$ such that $F_{+}^{-1}(0)=-\infty$. For instance, these would be the case if $\mathcal{M}=\mathcal{M}_{0}$. 


\section{Shrinking the set of transforms}

In this section, we discuss three issues related to the sets $\mathcal{G}^{L}$ and $\mathcal{G}^{R}$ used in formulating left- or right-ordinal covariance. The general goal is to understand whether a smaller set of transforms is sufficient for the result in Theorem 2. First, we show that, if the domain $\mathcal{M}$ is the set $\mathcal{M}_{c}$ of compacted supported distributions, then these sets can be slightly shrunk to $\mathcal{G}_{1}^{L}$ and $\mathcal{G}_{1}^{R}$ defined below, respectively. Second, we illustrate, by means of two counter-examples, that this relaxation does not work for general $\mathcal{M}$, and it is difficult to further make $\mathcal{G}_{1}^{L}$ and $\mathcal{G}_{1}^{R}$ smaller for $\mathcal{M}=\mathcal{M}_{c}$. Third, we provide an improvement of Chambers (2009) by shrinking the set used in his strong ordinal covariance property to $\mathcal{G}^{*}$.

Denote by $\mathcal{G}_{1}^{L}$ the set of all increasing left-continuous functions $\phi: \mathbb{R} \rightarrow \mathbb{R}$ satisfying

$$
\lim _{x \rightarrow-\infty} \phi(x)=-\infty \text { and } \lim _{x \rightarrow \infty} \phi(x)=\infty
$$

and by $\mathcal{G}_{1}^{R}$ the set of all increasing right-continuous functions $\phi: \mathbb{R} \rightarrow \mathbb{R}$ satisfying (7). It is clear that condition $(7)$ corresponds to $\phi(\mathbb{R})=\mathbb{R}$ in Definition 2 when $\phi$ is continuous. Therefore, $\mathcal{G}^{*} \subsetneq \mathcal{G}_{1}^{L} \subsetneq \mathcal{G}^{L}$ and $\mathcal{G}^{*} \subsetneq \mathcal{G}_{1}^{R} \subsetneq \mathcal{G}^{R}$.

Definition 3. A mapping $\rho: \mathcal{M} \rightarrow \mathbb{R}$ satisfies weak left-ordinal covariance (resp. weak rightordinal covariance) if $\rho \circ T^{[\phi]}=\phi \circ \rho$ for all $\phi \in \mathcal{G}_{1}^{L}\left(\right.$ resp. $\left.\phi \in \mathcal{G}_{1}^{R}\right)$.

Proposition 1. Let $\rho: \mathcal{M}_{c} \rightarrow \mathbb{R}$. We have

(i) $\rho$ satisfies weak left-ordinal covariance if and only if there exists $p \in(0,1]$ such that $\rho(F)=$ $F^{-1}(p)$ for all $F \in \mathcal{M}_{c}$;

(ii) $\rho$ satisfies weak right-ordinal covariance if and only if there exists $p \in[0,1)$ such that $\rho(F)=F_{+}^{-1}(p)$ for all $F \in \mathcal{M}_{c}$.

Proof. The "if" parts in both statements are implied by Theorem 2. We only show the "only if" parts.

(i) For any $F \in \mathcal{M}_{c}$, let

$$
\phi(x)=\left\{\begin{array}{cc}
x+F_{+}^{-1}(0), & x \leqslant 0 \\
F^{-1}(x), & 0<x \leqslant 1 \\
x-1+F^{-1}(1), & x>1 .
\end{array}\right.
$$

Then we have $\phi \in \mathcal{G}_{1}^{L}$ and $T^{[\phi]}\left(F_{U}\right)=F$, where $F_{U}$ is uniform on $[0,1]$. Hence,

$$
\rho(F)=\rho\left(T^{[\phi]}\left(F_{U}\right)\right)=\phi\left(\rho\left(F_{U}\right)\right) .
$$


Next we show $\rho\left(F_{U}\right) \in(0,1]$. We assume by contradiction that $\rho\left(F_{U}\right)>1$. For all $F \in \mathcal{M}_{c}$, $\rho(F)=F^{-1}(1)+a$ with $a=\rho\left(F_{U}\right)-1>0$. Letting $\varphi(x)=2 x, x \in \mathbb{R}$ and $F=\mathrm{U}[-1,0]$, we have

$$
\rho\left(T^{[\varphi]}(F)\right)=\left(T^{[\varphi]}(F)\right)^{-1}(1)+a=a .
$$

Moreover, by weak left-ordinal covariance, $\rho\left(T^{[\varphi]}(F)\right)=\varphi(\rho(F))=2 a$, implying $a=2 a=0$. Hence $\rho\left(F_{U}\right) \leqslant 1$. Next we assume $\rho\left(F_{U}\right) \leqslant 0$. Then for any $F \in \mathcal{M}_{c}, \rho(F)=F_{+}^{-1}(0)+\rho\left(F_{U}\right)$. For $\varphi(x)=x \mathbb{1}_{\{x \leqslant 0\}}+\left(1-\rho\left(F_{U}\right)+x\right) \mathbb{1}_{\{x>0\}} \in \mathcal{G}_{1}^{L}$, we have

$$
\rho\left(T^{[\varphi]}\left(F_{U}\right)\right)=\left(T^{[\varphi]}\left(F_{U}\right)\right)_{+}^{-1}(0)+\rho\left(F_{U}\right)=1>\rho\left(F_{U}\right)=\varphi\left(\rho\left(F_{U}\right)\right)=\rho\left(T^{[\varphi]}\left(F_{U}\right)\right),
$$

which is a contradiction. Hence, $\rho\left(F_{U}\right)>0$. It follows from (8), (9) and $\rho\left(F_{U}\right) \in(0,1]$ that $\rho(F)=F^{-1}\left(\rho\left(F_{U}\right)\right), \quad F \in \mathcal{M}_{c}$.

(ii) For any $F \in \mathcal{M}_{c}$, let

$$
\phi(x)=\left\{\begin{array}{cc}
x+F_{+}^{-1}(0), & x<0 \\
F_{+}^{-1}(x), & 0 \leqslant x<1 \\
x-1+F^{-1}(1), & x \geqslant 1 .
\end{array}\right.
$$

Then we have $\phi \in \mathcal{G}_{1}^{R}$ and $T^{[\phi]}\left(F_{U}\right)=F$. Hence, it follows that $\rho(F)=\rho\left(T^{[\phi]}\left(F_{U}\right)\right)=\phi\left(\rho\left(F_{U}\right)\right)$. Analogously to the proof of (i), we have $\rho\left(F_{U}\right) \in[0,1)$.

As a consequence of Proposition 1 and the fact that quantiles are monotone and semicontinuous, we immediately arrive at the following corollary.

Corollary 1. For mappings from $\mathcal{M}_{c}$ to $\mathbb{R}$,

(i) weak left-ordinal covariance implies monotonicity and lower seimicontinuity;

(ii) weak right-ordinal covariance implies monotonicity and upper seimicontinuity.

The next two counter-examples illustrate the subtle technical point that the choice of sets in both Theorem 2 and Proposition 1 cannot be easily made smaller. We first show that weak left- or right-ordinal covariance is not sufficient to guarantee that $\rho: \mathcal{M}_{0} \rightarrow \mathbb{R}$ is a quantile, and hence the assumption in Proposition 1 is not sufficient for the conclusion in Theorem 2.

Example 2. We divide the set $\mathcal{M}_{0}$ into four disjoint sets as follows:

$$
\begin{aligned}
& \mathcal{M}^{1}=\left\{F \in \mathcal{M}_{0}: F_{+}^{-1}(0)=-\infty \text { and } F^{-1}(1)<\infty\right\}, \\
& \mathcal{M}^{2}=\left\{F \in \mathcal{M}_{0}: F_{+}^{-1}(0)>-\infty \text { and } F^{-1}(1)=\infty\right\}, \\
& \mathcal{M}^{3}=\left\{F \in \mathcal{M}_{0}: F_{+}^{-1}(0)=-\infty \text { and } F^{-1}(1)=\infty\right\},
\end{aligned}
$$


and $\mathcal{M}^{4}=\mathcal{M}_{c}$. It follows that for $\phi \in \mathcal{G}_{1}^{L}, T^{[\phi]}\left(\mathcal{M}^{i}\right) \subseteq \mathcal{M}^{i}, i=1,2,3,4$. For $p_{1}, \ldots, p_{4} \in(0,1)$, define

$$
\rho(F)= \begin{cases}F^{-1}\left(p_{1}\right), & F \in \mathcal{M}^{1} \\ F^{-1}\left(p_{2}\right), & F \in \mathcal{M}^{2} \\ F^{-1}\left(p_{3}\right), & F \in \mathcal{M}^{3} \\ F^{-1}\left(p_{4}\right), & F \in \mathcal{M}^{4} .\end{cases}
$$

For $\phi \in \mathcal{G}_{1}^{L}$, using the closeness of $T^{[\phi]}$ on each $\mathcal{M}^{i}$ for $i=1,2,3,4$, we have $\rho\left(T^{[\phi]}(F)\right)=$ $\phi(\rho(F))$. If $p_{1}, \ldots, p_{4}$ are not identical, $\rho$ is not a quantile function on $\mathcal{M}_{0}$.

Our next question is whether $\mathcal{G}_{1}^{L}$ can be further made smaller to characterize quantiles on $\mathcal{M}_{c}$. A natural candidate might be $\mathcal{G}_{2}^{L}=\left\{\phi \in \mathcal{G}_{1}^{L}: \phi\right.$ is strictly increasing $\}$. Note that $\mathcal{G}^{*} \subsetneq \mathcal{G}_{2}^{L} \subsetneq \mathcal{G}_{1}^{L} \subsetneq \mathcal{G}^{L}$. In the next counter-example, we find that $\mathcal{G}_{2}^{L}$ is indeed not sufficient to force $\rho$ to be a quantile.

Example 3. Let us start with a split of $\mathcal{M}_{c}$. Define

$$
\mathcal{M}^{(1)}=\left\{F \in \mathcal{M}_{c}: F^{-1}(t) \text { is strictly increasing on }(0,1)\right\} \text {, and } \mathcal{M}^{(2)}=\mathcal{M}_{c} \backslash \mathcal{M}^{(1)}
$$

Note that for $\phi \in \mathcal{G}_{2}^{L}$,

$$
\left(T^{[\phi]}(F)\right)^{-1}(p)=\phi\left(F^{-1}(p)\right), p \in(0,1) .
$$

Hence for $\phi \in \mathcal{G}_{2}^{L}, T^{[\phi]}$ is closed on $\mathcal{M}^{(1)}$ and $\mathcal{M}^{(2)}$ respectively, i.e., $T^{[\phi]}\left(\mathcal{M}^{(i)}\right) \subseteq \mathcal{M}^{(i)}, i=1,2$. Define, for some distinct $p, q \in(0,1)$,

$$
\rho(F)= \begin{cases}F^{-1}(p), & F \in \mathcal{M}^{(1)} \\ F^{-1}(q), & F \in \mathcal{M}^{(2)} .\end{cases}
$$

Using the closeness of $T^{[\phi]}$ on $\mathcal{M}^{(1)}$, for $\phi \in \mathcal{G}_{2}^{L}$ and $F \in \mathcal{M}^{(1)}$,

$$
\rho\left(T^{[\phi]}(F)\right)=\left(T^{[\phi]}(F)\right)^{-1}(p)=\phi\left(F^{-1}(p)\right)=\phi(\rho(F)) .
$$

Moreover, using the closeness of $T^{[\phi]}$ on $\mathcal{M}^{(2)}$, for $\phi \in \mathcal{G}_{2}^{L}$ and $F \in \mathcal{M}^{(2)}$,

$$
\rho\left(T^{[\phi]}(F)\right)=\left(T^{[\phi]}(F)\right)^{-1}(q)=\phi\left(F^{-1}(q)\right)=\phi(\rho(F)) .
$$

Hence, $\rho$ satisfies $\rho \circ T^{[\phi]}=\phi \circ \rho$ for $\phi \in \mathcal{G}_{2}^{L}$. Nevertheless, $\rho$ is not a quantile.

On $\mathcal{M}=\mathcal{M}_{0}$, Chambers (2009) obtained a characterization of quantiles using a property called strong ordinal covariance, which replaces the set $\mathcal{G}^{*}$ in ordinal covariance by the larger set of strictly increasing and continuous functions $\phi: \mathbb{R} \rightarrow[-\infty, \infty]$ and $\mathbb{R} \subseteq \phi(\mathbb{R})$; in other words, $\pm \infty$ is allowed in the range of the functions $\phi$. In the next proposition, we show that $\mathcal{G}^{*}$ is sufficient for the desired result, thus relaxing the condition in Theorem 2 of Chambers (2009). 
Proposition 2. Let $\rho: \mathcal{M}_{0} \rightarrow \mathbb{R}$. We have

(i) $\rho$ satisfies monotonicity, ordinal covariance, and lower semicontinuity if and only if there exists $p \in(0,1)$ such that $\rho(F)=F^{-1}(p)$ for all $F \in \mathcal{M}_{0}$;

(ii) $\rho$ satisfies monotonicity, ordinal covariance, and upper semicontinuity if and only if there exists $p \in(0,1)$ such that $\rho(F)=F_{+}^{-1}(p)$ for all $F \in \mathcal{M}_{0}$.

Proof. We first show (ii) and then return to (i).

(ii) The "if" part is implied by Theorem 2 of Chambers (2009). We next focus on the "only if" part. As in Example 2, we divide $\mathcal{M}_{0}$ into four disjoint subsets $\mathcal{M}^{i}, i=1, \ldots, 4$. It follows from Theorem 1 of Chambers (2009) that there exists $p \in[0,1)$ such that

$$
\rho(F)=F_{+}^{-1}(p) \text { for all } F \in \mathcal{M}^{4}=\mathcal{M}_{c} .
$$

Next we extend this relation from $\mathcal{M}_{c}$ to $\mathcal{M}_{0}$ and show that $p>0$.

We first consider the distributions on $\mathcal{M}^{1}$. Define $F_{n}(x)=F(x) \mathbb{1}_{\{x \geqslant-n\}}, n \geqslant 1$. Note that $F_{n} \in \mathcal{M}_{c}$ and $F \leqslant_{\text {st }} F_{n}$. By monotonicity and (10), we have

$$
\rho(F) \leqslant \rho\left(F_{n}\right)=\left(F_{n}\right)_{+}^{-1}(p) .
$$

If $p=0$, then

$$
\rho(F) \leqslant \lim _{n \rightarrow \infty}\left(F_{n}\right)_{+}^{-1}(p)=\lim _{n \rightarrow \infty}-n=-\infty,
$$

which contradicts with the assumption that $\rho(F) \in \mathbb{R}$. Hence, $\rho \in(0,1)$. As a consequence, for $n$ sufficiently large, we have

$$
\rho(F) \leqslant\left(F_{n}\right)_{+}^{-1}(p)=F_{+}^{-1}(p) .
$$

As $F_{n}$ weakly converges to $F$ and $\rho\left(F_{n}\right)=F_{+}^{-1}(p)$ for sufficiently large $n$, by upper semicontinuity we obtain $\rho(F) \geqslant F_{+}^{-1}(p)$. Consequently, $\rho(F)=F_{+}^{-1}(p)$ for all $F \in \mathcal{M}^{1}$.

Next we focus on $\mathcal{M}^{2}$. Define $G_{n}(x)=F(x) \mathbb{1}_{\{x<n\}}+\mathbb{1}_{\{x \geqslant n\}}, n \geqslant 1$. We have $G_{n} \in \mathcal{M}_{c}$ and $G_{n} \leqslant$ st $F$. Hence, it follows from monotonicity and (10) that $\rho(F) \geqslant \rho\left(G_{n}\right)=\left(G_{n}\right)_{+}^{-1}(p)$. As $n$ sufficiently large, we have $\left(G_{n}\right)_{+}^{-1}(p)=F_{+}^{-1}(p)$, leading to $\rho(F) \geqslant F_{+}^{-1}(p)$. For $x_{0} \in \mathbb{R}$, we denote

$$
\phi_{n}(x)=\left\{\begin{array}{cc}
x, & x \leqslant x_{0} \\
x_{0}+\frac{x-x_{0}}{n}, & x>x_{0}
\end{array} \text {, and } \phi_{0}(x)=\min \left(x, x_{0}\right) .\right.
$$

Then it follows that $F \circ\left(\phi_{0}\right)_{+}^{-1} \in \mathcal{M}_{c}, F \circ\left(\phi_{n}\right)^{-1} \in \mathcal{M}^{2}$ and $F \circ\left(\phi_{0}\right)_{+}^{-1} \leqslant$ st $F \circ\left(\phi_{n}\right)^{-1}$ for $n \geqslant 1$. By ordinal covariance, we have

$$
\rho\left(F \circ\left(\phi_{n}\right)^{-1}\right)=\phi_{n}(\rho(F)) \geqslant \phi_{0}(\rho(F)) .
$$


Note that $F \circ\left(\phi_{n}\right)^{-1}$ weakly converges to $F \circ\left(\phi_{0}\right)_{+}^{-1}$. Hence, by upper semicontinuity, $\rho(F \circ$ $\left.\left(\phi_{0}\right)_{+}^{-1}\right) \geqslant \phi_{0}(\rho(F))$, which, together with $(10)$, implies $\phi_{0}\left(F_{+}^{-1}(p)\right) \geqslant \phi_{0}(\rho(F))$. If we take $x_{0}>\max \left\{F_{+}^{-1}(p), \rho(F)\right\}$, then the above equality implies $\rho(F) \leqslant F_{+}^{-1}(p)$. Consequently, $\rho(F)=$ $F_{+}^{-1}(p)$ for $F \in \mathcal{M}^{2}$.

We finally consider the distributions on $\mathcal{M}^{3}$. For $F \in \mathcal{M}^{3}$, let $F_{n}(x)=F(x) \mathbb{1}_{\{x \geqslant-n\}}, n \geqslant$ 1 , and $G_{n}(x)=F(x) \mathbb{1}_{\{x<n\}}+\mathbb{1}_{\{x \geqslant n\}}, n \geqslant 1$. Then we have $F_{n} \in \mathcal{M}^{2}, G_{n} \in \mathcal{M}^{1}$ and $G_{n} \leqslant_{\text {st }} F \leqslant$ st $F_{n}$. By monotonicity, $\rho\left(G_{n}\right) \leqslant \rho(F) \leqslant \rho\left(F_{n}\right)$. Using (10) on $\mathcal{M}^{1}$ and $\mathcal{M}^{2}$, we have

$$
\left(G_{n}\right)_{+}^{-1}(p) \leqslant \rho(F) \leqslant\left(F_{n}\right)_{+}^{-1}(p) .
$$

Letting $n \rightarrow \infty$ in the above inequalities, we obtain $\rho(F)=F_{+}^{-1}(p)$. This completes the proof of (ii).

(i) Define $\widehat{\rho}: \mathcal{M}_{0} \rightarrow \mathcal{M}_{0}$ by $\widehat{\rho}(F)=-\rho(\hat{F})$, where $\hat{F}$ the distribution of $-X$ for $X \sim F$. Then one can check that $\widehat{\rho}$ satisfies monotonicity, upper semicontinuity and ordinal covariance. Hence by (i) there exists $p \in(0,1)$ such that for all $F \in \mathcal{M}_{0}, \widehat{\rho}(F)=F_{+}^{-1}(p)$, which means that $\rho(\hat{F})=\hat{F}^{-1}(1-p)$. We complete the proof by noting that $\left\{\hat{F}: F \in \mathcal{M}_{0}\right\}=\mathcal{M}_{0}$.

Combination of Proposition 2 above and the results of Chambers (2009), we arrive at the following conclusion without imposing semicontinuity.

Corollary 2. Let $\rho: \mathcal{M} \rightarrow \mathbb{R}$ with $\mathcal{M}=\mathcal{M}_{0}$ or $\mathcal{M}=\mathcal{M}_{c}$. Then $\rho$ satisfies monotonicity and ordinal covariance if and only of there exists $p \in[0,1]$ such that $\rho(F)=F_{+}^{-1}(p)$ for all $F \in \mathcal{M}$ or $\rho(F)=F^{-1}(p)$ for all $F \in \mathcal{M}$.

\section{$5 \quad$ Locality and $\Lambda$-quantiles}

Next, we connect our results to the locality property, which intuitively means that the value of $\rho$ does not change if a distribution $F$ is replaced by a different one but identical to $F$ in a neighbourhood of $\rho(F)$. Locality is closely related to the pivotal monotonicity axiom of Rostek (2010) in decision theory. This property is satisfied by the quantiles, and more generally, they are satisfied by the $\Lambda$-quantiles introduced by Frittelli et al. (2014). For a function $\Lambda: \mathbb{R} \rightarrow[0,1]$, the $\Lambda$-quantile is defined as

$$
\rho(F)=\inf \{x \in \mathbb{R}: F(x) \geqslant \Lambda(x)\}
$$

Definition 4. For $\rho: \mathcal{M} \rightarrow \mathbb{R}$,

(i) $\rho$ is local if for any interval $(a, b) \subseteq \mathbb{R}, \rho(F) \in(a, b)$ implies $\rho(F)=\rho(G)$ for all $G \in \mathcal{M}$ with $G=F$ on $(a, b)$; 
(ii) $\rho$ is cash additive if $\rho\left(T^{[+c]} \circ F\right)=\rho(F)+c$ for all $F \in \mathcal{M}$ and $c \in \mathbb{R}$, where $T^{[+c]}=T^{[\phi]}$ with $\phi: x \mapsto x+c$;

(iii) $\rho$ is normalized if $\rho\left(\delta_{c}\right)=c$ for all $c \in \mathbb{R}$, where $\delta_{c}$ is a point mass at $c$.

We briefly verify that these three properties are weaker than left- or right-ordinal covariance. Cash additivity is implied by left- or right-ordinal covariance since $\phi: x \mapsto x+c$ for $c \in \mathbb{R}$ is in $\mathcal{G}^{L} \cap \mathcal{G}^{R}$, and normalization is implied by left- or right-ordinal covariance since $\phi: x \mapsto c$ for $c \in \mathbb{R}$ is also in $\mathcal{G}^{L} \cap \mathcal{G}^{R}$. Moreover, the function $\phi: x \mapsto a \vee x \wedge b$ for $a<b$ is in $\mathcal{G}^{L} \cap \mathcal{G}^{R}$. Note that $T^{[\phi]}(F)=T^{[\phi]}(G)$ if $F=G$ on $(a, b)$. Using left- or right-ordinal covariance we obtain $\phi(\rho(F))=\phi(\rho(G))$, and hence locality holds. Therefore, all three properties are implied by leftor right-ordinal covariance.

Locality is an essential property that leads to a characterization of $\Lambda$-quantile as shown by Bellini and Peri (2021). Since locality is weaker than left- or right-ordinal covariance, it remains to understand with what extra conditions quantiles can be characterized via locality. Theorem 13 of Bellini and Peri (2021) states that if $\rho: \mathcal{M}_{0} \rightarrow \mathbb{R}$ is normalized, monotone, lower semicontinuous and local, then $\rho$ is a $\Lambda$-quantile defined in (11) with a decreasing $\Lambda$. It turns out that if we include the extra property of cash additivity, then we arrive at a characterization of quantiles.

Theorem 3. For $\rho: \mathcal{M} \rightarrow \mathbb{R}$ with $\mathcal{M}=\mathcal{M}_{0}$ or $\mathcal{M}=\mathcal{M}_{c}$,

(i) $\rho$ is normalized, monotone, lower semicontinuous, cash additive and local if and only if there exists $p \in(0,1]$ such that $\rho(F)=F^{-1}(p)$ for all $F \in \mathcal{M}$;

(ii) $\rho$ is normalized, monotone, upper semicontinuous, cash additive and local if and only if there exists $p \in[0,1)$ such that $\rho(F)=F_{+}^{-1}(p)$ for all $F \in \mathcal{M}$.

Proof. (i) The "if" part follows directly from the properties of left-quantile. We will show the "only if" part. By Theorem 13 of Bellini and Peri (2021), $\rho$ is a $\Lambda$-quantile in (11) for some decreasing $\Lambda: \mathbb{R} \rightarrow[0,1]$. We next show that $\Lambda$ is a constant on $\mathbb{R}$. We assume by contradiction that there exist $x_{1}<x_{2}$ such that $\Lambda\left(x_{1}\right)>\Lambda\left(x_{2}\right)$. Let

$$
F_{1}(x)=\frac{\Lambda\left(x_{1}\right)+\Lambda\left(x_{2}\right)}{2} \mathbb{1}_{\left\{x_{1}-1 \leqslant x<x_{2}+1\right\}}+\mathbb{1}_{\left\{x \geqslant x_{2}+1\right\}}, x \in \mathbb{R} .
$$

By (11), we have $\rho\left(F_{1}\right) \in\left(x_{1}-1 / 2, x_{2}+1 / 2\right)$. Note that $T^{[+1 / 2]}\left(F_{1}\right)=F_{1}$ on $\left(x_{1}-1 / 2, x_{2}+1 / 2\right)$. Hence, locality implies $\rho\left(T^{[+1 / 2]}\left(F_{1}\right)\right)=\rho\left(F_{1}\right)$. However, it follows from cash additivity that

$$
\rho\left(T^{[+1 / 2]}\left(F_{1}\right)\right)=\rho\left(F_{1}\right)+\frac{1}{2},
$$

leading to a contradiction. Therefore, $\Lambda$ is a constant on $\mathbb{R}$ which is denoted by $p \in[0,1]$. Hence by (11), we have $p \in(0,1]$ and $\rho(F)=F^{-1}(p)$. Note that $p \in(0,1)$ if $\mathcal{M}=\mathcal{M}_{0}$. 
The proof of (ii) is similar to that of (i) and is omitted.

Similarly to Remark 1 , in case $\mathcal{M}=\mathcal{M}_{0}$, the value $p=1$ in (i) and the value $p=0$ in (ii) need to be excluded. A direct consequence of the above result and Theorem 2 is a relation between left- or right-ordinal covariance and locality.

Corollary 3. For mappings from $\mathcal{M}_{c}$ or $\mathcal{M}_{0}$ to $\mathbb{R}$,

(i) left-ordinal covariance is equivalent to normalization, monotonicity, lower semicontinuity, cash additivity and locality together;

(ii) right-ordinal covariance is equivalent to normalization, monotonicity, upper semicontinuity, cash additivity and locality together.

\section{Acknowledgments}

RW acknowledges financial support from the Natural Sciences and Engineering Research Council of Canada (RGPIN-2018-03823, RGPAS-2018-522590).

\section{References}

Bellini, F. and Peri, I. (2021). An axiomatization of $\Lambda$-quantiles. arXiv: 2109.02360.

Chambers, C. P. (2007). Ordinal aggregation and quantiles. Journal of Economic Theory, 137(1), $416-431$.

Chambers, C. P. (2009). An axiomatization of quantiles on the domain of distribution functions. Mathematical Finance, 19(2), 335-342.

de Castro, L. and Galvao, A. F. (2019). Dynamic quantile models of rational behavior, Econometrica, 87, 1893-1939.

de Castro, L. I., Galvao, A. F., Noussair, C. and Qiao, L. (2020). Do people maximize quantiles? SSRN : 3607612 .

Embrechts, P., Liu, H. and Wang, R. (2018). Quantile-based risk sharing. Operations Research, 66(4), 936-949.

Föllmer, H. and Schied, A. (2016). Stochastic Finance. An Introduction in Discrete Time. Walter de Gruyter, Berlin, Fourth Edition.

Frittelli, M., Maggis, M., and Peri, I. (2014). Risk measures on $\mathcal{P}(\mathbb{R})$ and Value at Risk with probability/loss function. Mathematical Finance, 24(3), 442-463.

Gneiting, T. (2011). Making and evaluating point forecasts. Journal of the American Statistical Association, 106(494), 746-762. 
He, X. and Peng, X. (2018). Surplus-invariant, law-invariant, and conic acceptance sets must be the sets induced by Value-at-Risk. Operations Research, 66(5), 1268-1276.

Liu, F. and Wang, R. (2021). A theory for measures of tail risk. Mathematics of Operations Research, 46(3), 1109-1128.

Liu, P. and Schied, A. and Wang, R. (2021). Distributional transforms, probability distortions, and their applications. Mathematics of Operations Research, published online.

Koenker, R. and Hallock, K. F. (2001). Quantile regression. Journal of Economic Perspectives, 15(4), 143-156.

Kou, S. and Peng, X. (2016). On the measurement of economic tail risk. Operations Research, 64(5), 1056-1072.

McNeil, A. J., Frey, R. and Embrechts, P. (2015). Quantitative Risk Management: Concepts, Techniques and Tools. Revised Edition. Princeton, NJ: Princeton University Press.

Rostek, M. (2010). Quantile maximization in decision theory. Review of Economic Studies, 77, $339-371$.

Wang, R. and Wei, Y. (2020). Risk functionals with convex level sets. Mathematical Finance, 30(4), 1337-1367.

Wang, R. and Zitikis, R. (2021). An axiomatic foundation for the Expected Shortfall. Management Science, 67, 1413-1429. 